\title{
DESENVOLVIMENTO DE PROTÓTIPO DE UMA BENGALA ULTRASSÔNICA UTILIZANDO PLATAFORMA ARDUINO
}

\author{
Douglas Alves de Sousa (FIC/UNIS) - douglas.sousa@alunos.unis.edu.br \\ Fabiano Dias Moreira (FIC/UNIS) - fabiano.moreira@alunos.unis.edu.br \\ Flávio Dias Moreira (FIC/UNIS) - flavio.moreira@alunos.uni.edu.br \\ Wesley Ciqueira de Oliveira (FIC/UNIS) - wesley.oliveira@alunos.unis.edu.br \\ Sanderson Rocha de Abreu - sanderson.abreu@unis.edu.br
}

\begin{abstract}
RESUMO: Este estudo aborda o desenvolvimento de um protótipo de uma bengala ultrassônica. Tal abordagem se faz necessária devido a relevância do projeto no auxílio para deficientes visuais. $O$ intento deste estudo é enfatizar como a locomoção e execução de atividades diárias dos portadores pode ser aprimorada com a evolução deste presente labor. Fundamentalmente, a bengala utiliza-se de ondas ultrassonicas para identificar objetos abaixo da linha da cintura do deficiente, impedindo-o de colidir com objetos presentes nesta posição quando ocorrer a locomoção. $O$ sensor acoplado à bengala emite ondas ultrassônica alternadas que, ao detectar obstáculos, são refletidas retornando ao sensor. Um microcontrolador recebe as informações do objeto, analisando o grau de perigo de colisão com o deficiente visual, acionando o buzzer, o qual emite um sinal sonoro, à medida que o deficiente se aproxima intensamente do objeto, maior será a frequência do som emitido do buzzer.
\end{abstract}

\section{INTRODUÇÃO}

No cenário mundial, segundo a Organização Mundial da Saúde (OMS, 2017), existem aproximadamente 39 milhões de pessoas portadoras de deficiência visual. Enquanto no Brasil, conforme dados estratificados do Instituto Brasileiro de Geografia e Estatística (IBGE, 2016), provindos de pesquisas realizadas no ano de 2015, inferem que $6 \%$ da população apresenta algum tipo de deficiência, sendo a visual de maior prevalência, com $3,6 \%$ da população, totalizando cerca de 432.000 pessoas.

A deficiência visual, também considerada como cegueira, é aquela na qual a acuidade visual é igual ou menor que 0,05 no melhor olho, com a melhor correção óptica; a baixa visão, que significa acuidade visual entre 0,3 e 0,05 no melhor olho, com a melhor correção óptica; os casos nos quais a somatória da medida do campo visual em ambos olhos for igual ou menor que $60^{\circ}$; ou a ocorrência simultânea de quaisquer das condições descritas. (DA PAZ, 2006)

Em concordância com as informações supracitadas, a idealização do projeto da bengala ultrassônica provém da necessidade da melhoria qualitativa rotineira dos deficientes visuais. O intento de ordem primaz se resume no auxílio das tarefas diárias tais como locomoção social, acessibilidade à locais com ressaltos e identificação de obstáculos por meio da informação sonora e vibrações detectadas pelo sensor e transferidas ao buzzer, o qual permite ao usuário distinguir a possível barreira existente.

O objetivo do presente trabalho é desenvolver um protótipo de uma bengala ultrassônica que é integrada de um sensor e um buzzer acoplados a uma bengala de madeira, para os deficientes visuais. O estudo para implementação do projeto foi realizado na cidade de Cataguases - Minas Gerais, a qual possui 4.031 portadores de deficiência visual. Os objetivos específicos do estudo consistem no desenvolvimento de uma bengala ultrassônica utilizando plataforma Arduino, através da programação desenvolvida no software. Após esse processamento, o código programado é passado para a memória do Arduino, e, por conseguinte, essa informação recebida é transferida 
ao sensor ultrassônico, cujo papel é elaborar a leitura da distância do usuário a um determinado objeto, através da emissão de ondas, como também ao buzzer, que emite um sinal sonoro de acordo com a diminuição da proximidade de um indivíduo. Quanto menor o espaço entre o objeto e o usuário, identificada pelo sensor, maior será a frequência emitida pelo buzzer.

\section{MATERIAIS E MÉTODOS}

Equipamentos utilizados na construção do projeto e suas especificações que estão disponíveis no mercado, como demostra a tabela 1.

Tabela 1: Descrição dos equipamentos para a montagem do protótipo

\begin{tabular}{|l|l|}
\hline Equipamentos & Descrição \\
\hline Arduino & UNO \\
\hline Bateria & $9 \mathrm{v}$ \\
\hline Bengala & Madeira Indaia \\
\hline Parafusos & Rosca soberba \\
\hline Abraçadeiras & Nylon \\
\hline Jumpers & Macho-macho e macho-fêmea \\
\hline Buzzer & Emissor sonoro \\
\hline
\end{tabular}

Fonte: Autores (2018)

A Tabela consta os equipamentos utilizados na realização do presente projeto, assim como suas respectivas características, sendo o Arduino UNO a peça principalparaaleituradocódigodaprogramaçãoetransmitidoparaosoutroscomponentesdosi stema.

O presente artigo foi desenvolvido através de uma pesquisa de campo por meio de projetos já elaborados. Analisados os números de deficientes visuais a âmbito mundial, nacional e na cidade de Cataguases, houve a necessidade de elaboração do presente labor. Baseado na lógica estrutural de artigos que discorreram sobre a importância da implementação e das dificuldades dos portadores de deficiência visual em locomoção, acessibilidade, foram analisados livros e artigos dispostos no acervo das Faculdades Integradas de Cataguases (FIC/UNIS). No banco de dados do Google Acadêmico foram consultados artigos científicos, sites de universidades federais, sites nacionais. A seleção dos materiais para a confecção deste trabalho foi realizada entre as datas de 14 de setembro a 15 de outubro de 2018.

A metodologia utilizada foi de natureza básica. De acordo com Oliveira (2011), este tipo de pesquisa tem por objetivo apresentar como se pretende realizar a investigação, descrever a classificação quanto aos objetivos da pesquisa, a natureza da pesquisa, o objeto a ser estudado, a técnica de coleta e a técnica de análise de dados.

O desenvolvimento do projeto compreende a utilização do programa Arduino na linguagem $\mathrm{C}$, onde está armazenado toda a sua funcionalidade. Após esta etapa, transfere- se todo o programa desenvolvido para um microcontrolador, cujo objetivo é fazer a leitura técnica das funções programadas e transmitir para os aparelhos acoplados às funções estabelecidas no programa.

O código da programação foi desenvolvido no IDE (Arduino Integrated Development Envirnoment) de maneira adaptativa, ou seja, originada a partir da análise 
sistemática de uma programação já estabelecida conforme o artigo realizado pelo grupo Unis, cuja função norteadora proporcionou modificações, adaptado do projeto de Arduino e Cia, conforme o apêndice A.

Para o desenvolvimento do protótipo foram utilizados materiais específicos adaptadosaoprojetoemquestão.Fez-seusodeumabengaladomodeloIndaiademadeira de 92 centímetros de extensão, Arduino Software em linguagem de programação C, microcontrolador arduino UNO, sensor ultrassônico HC-SRO4, gerador de sinal sonoro (Buzzer), jumpers macho-macho e macho-fêmea, bateria de 9 volts, demonstrados de acordo com a Figura 1.

Figura 1: Transferência do programa para a memória do Arduino tal qual sua alimentação feita pelo computador

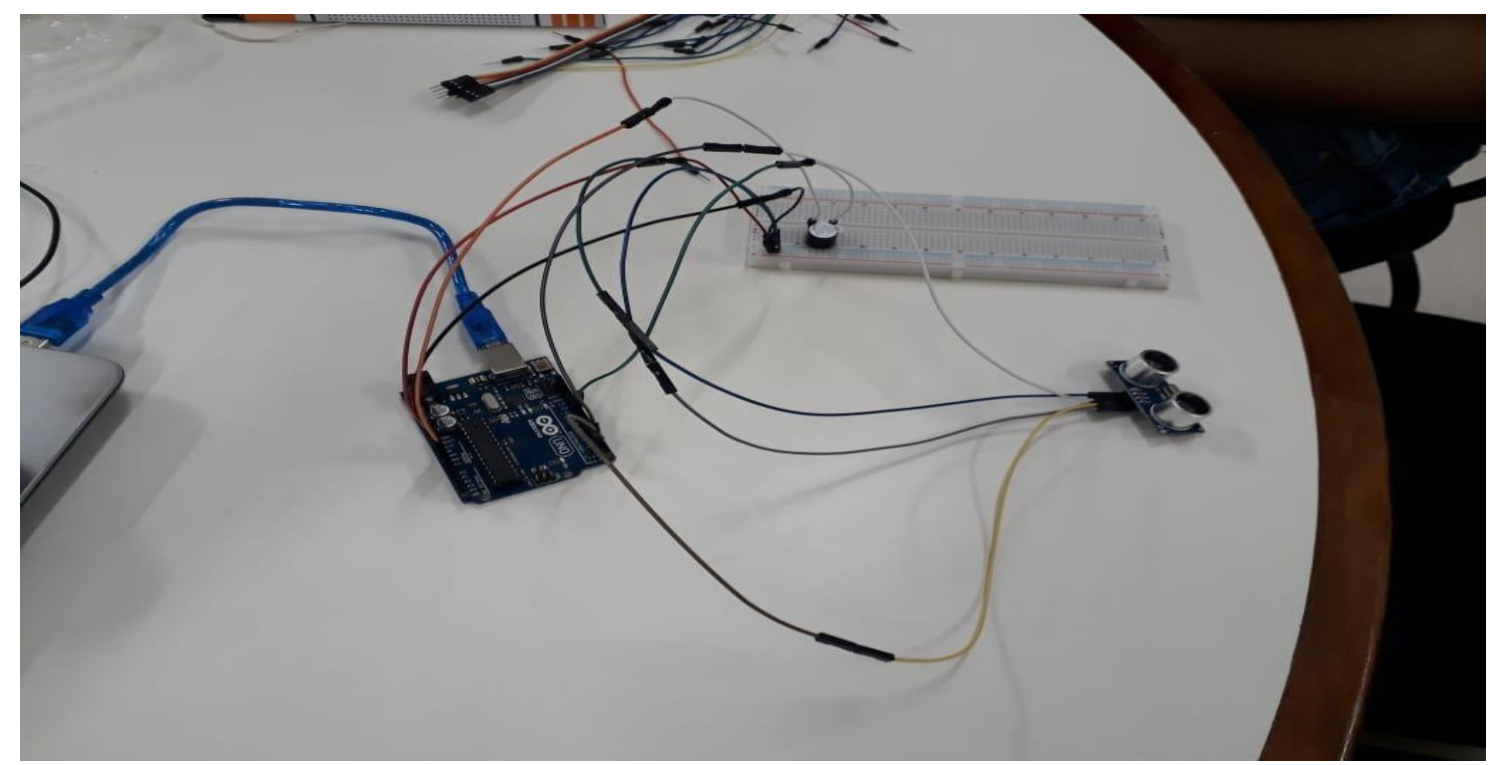

Fonte: Autores (2018)

Conforme a Figura 1, há a utilização dos jumpers macho-macho e macho-fêmea interligados entre si, os quais foram conectados ao sensor pelas portas GND e VCC, simultaneamente acopladas ao Arduino UNO pelos pinos VCC e GND. A conecção dos jumpers nos pinos Trigger (Emissor de ondas sonoras) e Echo (Receptor de ondas sonoras) do sensor às entradas Trigger (pino 3) e Echo (pino 2) no Arduino ocorrem sucessivamente. Logo, o passo conseguinte demonstra as conecções do VCC e GND do sensor na protoboard concomitantemente à conecção vinda do Arduino à placa protoboard e a ligação do buzzer. Na última etapa ocorre a conecção do cabo USB ao Arduino, servindo como fonte de alimentação, e também transferindo a programação feita no software para a memória do Arduino, conforme a Figura2. 
Figura 2: Sistema com o sensor, Arduino e buzzer

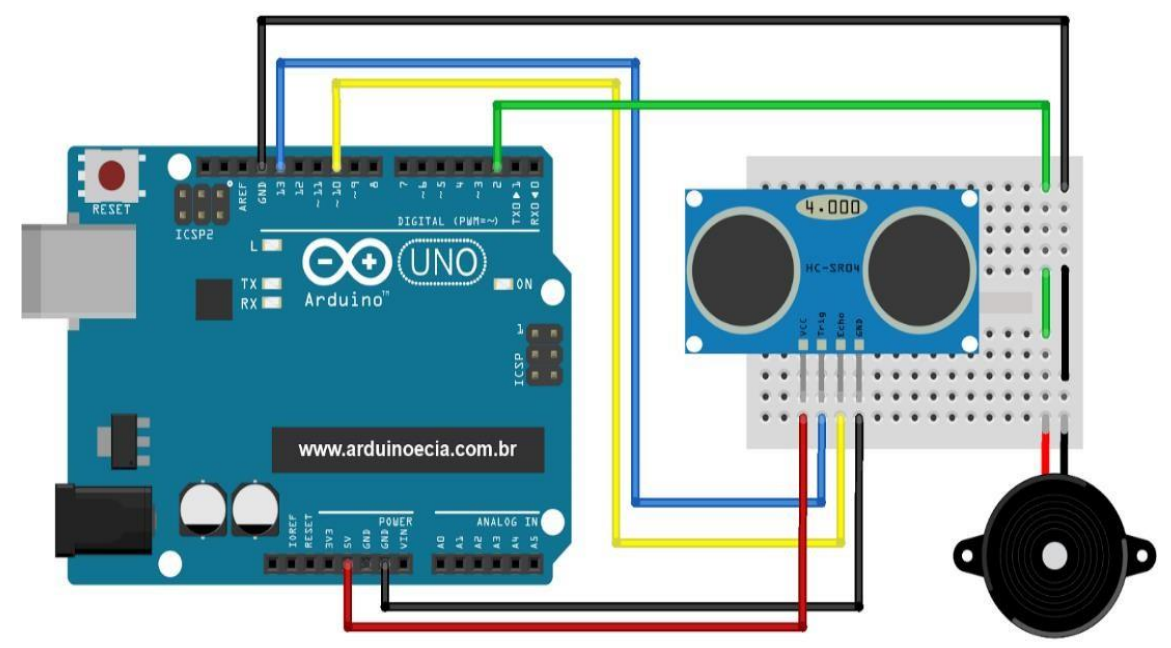

Fonte: Adaptado (Arduino e Cia)

De acordo com a Figura 2, é apresentado o circuito criado sobre a Protoboard e o microcontrolador com a ajuda do software Fritzing, para um melhor entendimento e compreensão, apresentando as ligações e os componentes utilizados para elaboração do protótipo.

\section{REFERENCIAL TEÓRICO}

\subsection{Prototipagem}

Implementação de controle de sistemas automatizados por meio de software, hardware e de Controladores Programáveis para Automação (PAC). Envolve a concepção de todo o projeto de um sistema de controle em automação por meio de ferramentas colaborativas, desde as etapas de modelagem, de simulação e de arquitetura de controlador, até sua implementação final em hardware dedicado. (ROSÁRIO, 2009).

\subsection{Tecnologia Assistiva}

De acordo com Bersch (2013), é uma área que abrange determinados aspectos relativos a métodos e equipamentos que são construídos por especialistas, pesquisadores que objetivam a melhora da qualidade de vida das pessoas com deficiência parcial ou total, sendo físicas e/ou intelectuais.

Segundo o Comitê de Ajudas Técnicas - CAT (2012):

Tecnologia assistiva é uma área do conhecimento, de característica interdisciplinar, que engloba produtos, recursos, metodologias, estratégias, práticas e serviços que objetivam promover a funcionalidade, relacionada à atividade e participação de pessoas com deficiência, incapacidades ou mobilidade reduzida, visando sua autonomia, independência, qualidade de vida e inclusão social" (ATA VII - Comitê de Ajudas Técnicas CAT2012).

\subsection{Legislação}

Todos os direitos das pessoas com deficiência são demonstrados em fóruns internacionais, políticas públicas e nas ações governamentais brasileiras. Em 1975, a Organização das Nações Unidas proclamou a Declaração dos Direitos da Pessoa Deficiente, conforme definido em seu $\operatorname{artigo}^{\circ}$ o conceito de pessoa deficiente: “ Qualquer pessoa incapaz de assegurar por si mesma, total ou parcialmente, as 
necessidades de uma vida individual ou social normal, em decorrência de uma deficiência congênita ou não, em suas capacidades físicas, sensoriais ou mentais".

A Política Nacional para a Integração da Pessoa Portadora de Deficiência compreende, em seu artigo $2^{\circ}$, assegurar à pessoa portadora de deficiência o pleno exercíciodeseusdireitosbásicos, inclusivedosdireitosàeducação,àsaúde, aotrabalho, ao desporto, ao turismo, ao lazer, à previdência social, à assistência social, ao transporte, à edificação pública, à habitação, à cultura, ao amparo à infância e à maternidade, e de outrosque,decorrentesdaConstituiçãoedasleis,propiciemseubem-estarpessoal,social e econômico, segundo a Lei $\mathrm{n}^{\mathrm{O}} 7.853$, de 24 de outubro de1989.

O preconceito torna-se criminalizado em razão da deficiência, além de instituir a Política Nacional para Integração da Pessoa Portadora de Deficiência. Em 1991, através de Lei 8.213/91, foi instaurada cotas para que as empresas privadas e órgãos públicos reservem vagas para pessoas com deficiência. Em 1999, o decreto 3298/99, regulamenta aLei7.853/89, quanto à conceituação de deficiência e parâmetro para avaliação de todos os tipos de deficiências, a reserva pode variar conforme o número de funcionários, com início de $2 \%$ para até 200 empregados, 3\% de 201 a 500, 4\% de 501 a 1.000 e 5\% para aquelas que possuem mais de 1.000. O artigo 93, prevê a proibição de qualquer ato discriminatório relacionado ao salário ou critério de admissão do emprego em virtude de possuir deficiência.

\subsection{Microcontrolador}

É um hardware com um alto poder de controle e menor custo benefício. Este

Hardware é o Microcontrolador que segundo Chase (2007, p. 6)

Os microcontroladores englobam em um único chip: interfaces de entrada/saída digitais e analógicas, periféricos importantes como a memória RAM memória FLASH, interfaces de comunicação serial, conversores analógico-digitais e temporizadores/contadores (CHASE, 2007, p.6).

\subsection{Arduino (Software)}

De acordo com Banzi (2014), o Arduino é composto por duas partes principais, sendo a segunda o Arduino Integrated Development Envirnoment, or IDE, a parte do software executada no computador. Usa-se o IDE para criar o sketch (pequeno programa computacional) que carrega a placa do Arduino. O sketch diz para a placa o que deve ser feito.

A linguagem $\mathrm{C}$ foi inventada e implementada primeiramente por Dennis Ritchie e um DEC PDP-11 que utilizava o sistema operacional UNIX. C é o resultado de processo de desenvolvimento que começou com uma linguagem mais antiga chamada BCPL, que ainda está em uso, em sua forma original na Europa. BCPL foi desenvolvida por Martin Richards e influenciou uma linguagem chamada B, inventada por Ken Thompson. Na década de 1970, B levou ao desenvolvimento de C. (HERBET SCHILDT, 1996, p. 1-3).

\subsection{Arduino UNO}

Segundo BLUM (2018), o Arduino é uma placa de controle de entrada de dados (IN), como sensores, e saída de dados (OUT), como motores e leds, com cristal oscilador de $16 \mathrm{Mhz}$, um regulador de tensão de $5 \mathrm{~V}$, botão de reset, plugue de alimentação, pinos conectores, e alguns LEDs para facilitar a verificação do 
funcionamento. Aporta USB já fornece alimentação enquanto estiver conectado ao computador, e a tensão de alimentação quando desconectado pode variar de $7 \mathrm{~V}$ a12V, graças à o regulador presente na placa. No Arduino, informações ou ordens são transmitidas de um computador para a placa através de Bluetooth, wireless, USB, infravermelho, etc. Essas informações devem ser traduzidas utilizando a linguagem Wiring baseada em $\mathrm{C} / \mathrm{C}++$.

\subsection{Sensor Ultrassônico (HC-SRO4)}

Segundo a Universidade Estadual Paulista (2010), um oscilador emite ondas ultrassônicas (emtornode $42 \mathrm{kHz}$ ), que resultam em um comprimento de onda de alguns centímetros, o que permite detectar objetos relativamente pequenos.

As ondas refletidas pelo objeto são captadas pelo sensor, fornecendo um sinal que pode ser processado trazendo informações sobre o objeto no qual ocorreu a reflexão.

O HC-SRO4 utiliza sinais ultrassônicos (40 Khz, acima da capacidade de audição do ouvido humano, que é de20Khz), para determinar a distância entre o sensor e o obstáculo, ele pode medir distâncias entre $2 \mathrm{~cm}$ e $4 \mathrm{~m}$, com precisão de $3 \mathrm{~mm}$, seu ângulo de detecção é de aproximadamente 15 graus, segundo informações do datasheet 14 do sensor. Seu funcionamento consiste basicamente em enviar um sinal que, ao atingir um objeto, volta para o sensor e com base nesse tempo entre o envio e recebimento, é calculada a distância entre o sensor e o objeto. (THOMSEN,2015).

\subsection{Buzzer}

É um componente eletrônico composto de uma membrana vibratória em um invólucro, muitas vezes plástico. Ao excitá-lo eletricamente, a membrana vibra e produz ruído. Alternando a frequência de excitação, é possível conseguir alguns tons. (FRIZZARIN, 2014)

\section{RESULTADOS E DISCUSSÕES}

O trabalho visa a busca de alternativas para transformar qualitativamente a vida dos deficientes visuais por meio de tecnologias de fácil utilização, as quais contribuirão para a redução de acidentes auxiliando estes que enfrentam grandes dificuldades no que tange a mobilidade urbana e a inacessibilidade conspícua.

Figura 3: Bengala Ultrassônica

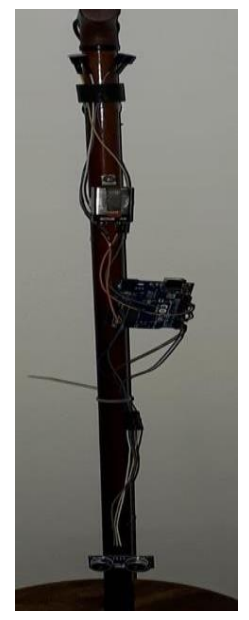


O projeto é fundamentado na tecnologia assistiva, cujo o intento é a utilização dos recursos tecnológicos a fim de obter melhorias na vida dos deficientes, usando ferramentas para o auxílio locomotivo, pois a transcorrência dos obstáculos é oriunda da falta de recursos básicos para segurança pública, tais como pavimentação e sinalização adequadas.

A bengala funciona como um radar. O sensor, acoplado em sua parte inferior, emite ondas eletromagnéticas através do pino Trigger, as quais se chocam contra o objeto produzindo uma resposta, que recebida pelo pino Echo, volta para o sensor e com base nesse tempo entre o envio e recebimento, é calculada a distância entre o sensor e o objeto, sendo assim, acionando o buzzer, que, cada vez mais próximo se encontrar do objeto, emitirá maiores frequências de sinais sonoros, alertando o deficiente da distância dos obstáculos.

À medida que se aproxima dos obstáculos, essa resposta torna-se mais intensa aumentando a intensidade sonora. A bengala possui 1 sensor localizado na parte inferior da bengala a 5 centímetros ao ponto de contato do piso. O deslocamento da bengala de um lado para o outro garante a detecção de possíveis barreiras laterais e frontais. O sistema é alimentado por uma pilha de 9volts.

\section{CONCLUSÃO}

Com o expressivo número de deficientes no mundo, sendo aproximadamente 39 milhões de deficientes visuais e cerca de 432.000 pessoas no Brasil, torna-se imprescindível a criação do presente labor, haja vista as multifaces de utilização e os benefícios por ele gerado na vida desses deficientes. Com isso, o objetivo do projeto é desenvolver de forma sistêmica uma bengala ultrassônica com um sensor e um buzzer integrados, capaz da identificação de objetos por meio de ondas emitidas através do sensor.

O protótipo é ligado à uma bateria externa de 9 volts, cuja função é alimentar o sistema.Odesenvolvimentodabengalaultrassônicadeu-sepelousodeumArduinoUno cuja programação foi desenvolvida no IDE e posteriormente enviada à memória do Arduino. Através dos jumpers macho-macho e macho-fêmea conectou-se o sensor ultrassônico HC-SRO4 nos pinos Trigger e Echo, concomitantemente com o buzzer.

Paraafasedetestesforamestipuladosintervalosdedistânciaparaoacionamento do sensor e consequentemente do buzzer. O sensor ultrassônico tem capacidade de medição de aproximadamente 4 metros, conforme fabricante. A leitura das medições começa no primeiro intervalo quecompreendeadistânciade1,80metrosaté1,30metros com o delay de 700 Microssegundos para o buzzer acionado e 600 Microssegundos para o buzzer desligado, emitindo sinais sonoros com frequências maiores ao mesmo tempo em que a distância diminui. O último intervalo compreende a distância entre 0,89 metros e 0,50 metros, os quais a frequência aumentou consideravelmente. Caso a distância seja inferior a 0,50 metros, o buzzer emite um som com frequência contínua, possibilitando ao deficiente a identificação do obstáculo tal qual sua proximidade.

A principal contribuição deste trabalho foi criar um dispositivo que auxilia a locomoção de um deficiente visual através de um alerta de obstáculos, tal dispositivo é denominado bengala ultrassônica.

\section{REFERÊNCIAS BIBLIOGRÁFICAS}

Arduino e Cia, Sensor de Estacionamento/Ré com Arduino e Sensor Ultrassônico HC-SRO4. Disponível em: <https://www.arduinoecia.com.br/2014/04/sensor-de-estacionamento-re-comarduino.html>. (Acessoem: 15/10/2018) 
BANZI, Massimo and Shiloh, Michael. Getting Started with Arduino: The Open Source Electronics Prototyping Platform. Disponível em: < https://books.google.com.br/books?hl=pt-

BR\&lr=\&id=Xd3SBQAAQBAJ\&oi=fnd\&pg=PT21\&dq=Banzi\&ots=zZXafYkcVI\&sig=WtpDGMA 2hWxwSW2JrgLj6Bd5OSE\#v=onepage \&q\&f=false>. (Acesso em:14/08/2018)

BERSCH, Rita. Introdução à Tecnologia Assistiva. Disponível em: <http://www.assistiva.com.br/Introducao_Tecnologia_Assistiva.pdf>. (Acesso em: 15/10/2018) BLUM, Jeremy. Explorando o Arduino: Técnicas e Ferramentas para Mais Dicas de Engenharia. Ed.Alta Books Editora

BRASIL, Palácio do Planalto. Decreto $\mathrm{N}^{\mathrm{o}}$ 3.298/99. Brasília, DF, e < Disponível http://www.planalto.gov.br/ccivil_03/decreto/D3298.htm>. m

(Acessoem:21/12/2018)

BRASIL, Palácio do Planalto. Lei Federal 7.853/89. Brasília, DF, e < Disponível http://www.planalto.gov.br/ccivil_03/leis/17853.htm>. m

(Acessoem09/11/2018)

BRASIL, Palácio do Planalto. Lei Federal 8.213/91. Brasília, DF, Disponível e < http://www.planalto.gov.br/ccivil_03/leis/18213cons.htm> (Acesso em06/09/2018) m

CAT,2007a.AtadaReuniãoIII,deabrilde2007,ComitêdeAjudasTécnicas,SecretariaEspecialdos Direitos Humanos da Presidência da República (CORDE/SEDH/PR). (Acesso em:01/09/2018)

CHASE, Otavio. Sistemas Embarcados. Disponível em: <https://s3.amazonaws.com/academia.edu.documents/42673850/Embarcados.pdf?AWSAccessKeyId =AKIAIWOWYYGZ2Y53UL3A\&Expires=1540170930\&Signature=Ef1kKtjkjrbbmAaavl\%2Bi4G ABwsU \%3D\&response-content-disposition=inline\%3B\%20filename\%3DEmbarcados.pdf $>$. (Acesso em: 05/09/2018)

DA PAZ, RonilsonJosé. A Pessoas Portadoras de Deficiência no Brasil, Inclusão Social. Disponível em:

https://books.google.com.br/books?id=DQLiziFAxW4C\&pg=PA20\&dq=conceito+de+Defici\%C3\%A A ncia $+\mathrm{f} \% \mathrm{C} 3 \%$ ADsica+visual\&hl=pt-

$\mathrm{BR} \& \mathrm{sa}=\mathrm{X} \& \mathrm{ved}=0 \mathrm{ahUKEwjgne} 3 \mathrm{fydrg}$ AhUrFLkGHdhMDn4Q6AEIKDAA\#v=onepage \&q=conceito $\% 20$ de\%20Defici\%C3\%AAncia\%20f\%C3\%ADsica\%20visual\&f=false>. (Acesso em: 11/09/2018)

FRIZZARIN BRYAN, Fernando. Arduino: Guia para Colocar suas Ideias em Prática. Disponível em:

$<$

https://books.google.com.br/books?id=FcPXCwAAQBAJ\&pg=PT125\&dq=o+que+\%C3\%A9+um+ buzzer\&hl=pt-

BR\&sa $=$ X\&ved=0ahUKEwjCiqqKv93gAhVBHrkGHS0IAdIQ6AEILTAB\#v=onepage \&q $=0 \% 20 \mathrm{q}$ ue\%20\%C3\%A9\%20um\%20buzzer\&f=false>. (Acesso em: 18/10/2018)

INSTITUTO BRASILEIRO DE GEOGRAFIA E ESTATÍSTICA (IBGE). Disponível em: < https://www.ibge.gov.br/>. (Acesso em: 15/09/2018)

ONU. Declaração dos direitos das pessoas com deficiência. Assembleia Geral das Organizações $\begin{array}{llll}\text { das Nações Unidas } & \text { em } & \text { 09/12/1975. } & \text { Disponível }\end{array}$ em:

<http://portal.mec.gov.br/seesp/arquivos/pdf/dec_def.pdf>. (Acesso em: 01/10/2018)

OLIVEIRA, MAXWELL FERREIRA. Conceito de Metodologia Científica. Disponível em: <https://adm.catalao.ufg.br/up/567/o/Manual_de_metodologia_cientifica__Prof_Maxwell.pdf>. (Acesso em: 12/10/2018)

ORGANIZAÇÃO MUNDIAL DA SAÚDE (OMS). Disponível em: < http://www.who.int/eportuguese/countries/bra/pt/>. (Acesso em: 10/10/2018) 
ROSÁRIO MAURÍCIO, João. Automação Industrial. Disponível em: $<$ https://books.google.com.br/books?id=YsUHLcHdbh4C\&pg=PA68\&dq=conce ito+de+prot\%C3\%B3tipagem\&hl=pt-

$\mathrm{BR} \& \mathrm{sa}=\mathrm{X} \& \mathrm{ved}=0$ ahUKEwi6nKfvwt3gAhXmILkGHTY7CgEQ6AEILjAB\# $\mathrm{v}=$ onepage $\& \mathrm{q}=$ conceito $\% 20$ de\%20prot\%C3\%B3tipagem\&f=false>. (Acesso em:29/09/2018)

SCHILDT, Herbert. C Completo e Total. $3^{a}$ edição, revista e ampliada. São Paulo: Makron Books do Brasil, 1997. Pág.: 1-3. (Acesso em: 02/10/2018)

THOMSEN, Adilson. Como utilizar o sensor ultrassônico HC-SR04. Disponível em:

<http://buildbot.com.br/blog/como-utilizar-o-sensor-ultrasonico-hc-sr04/>. (Acesso em: 28/10/2018) UNIVERSIDADE ESTADUAL PAULISTA. Conceito de Sensor Ultrassônico. Disponível em: <http://www.joinville.udesc.br/portal/professores/saimon/materiais/Sensores.pdf〉. (Acesso em: 21/10/2018)

\section{APÊNDICE A - Programação}

\#define pEcho 2 //definições das entradas dos pinos no arduino.

\#define pTrig3

\#define buzz10

\#define buzzdois 6

unsignedlong tempo; //dando entrada nas

variaveis. double distancia;

void setup () \{ //entradas e saidas.

pinMode(pEcho, INPUT);

pinMode(pTrig, OUTPUT);

pinMode(buzz, OUTPUT);

pinMode(buzzdois, OUTPUT);

\}

digitalWrite(pTrig, LOW);

void $\quad \operatorname{loop}()\{$

//Acioname

nto. digitalWrite (pTrig, HIGH);

delayMicroseconds(10);

digitalWrite(pTrig,LOW);

tempo $=$ pulseIn $(\mathrm{pEcho}, \mathrm{HIGH}) ; / /$ captura da duração de um

pulso. distancia $=$ tempo/58;//conversão de tempo em distância.

aviso(distancia);

delay(100);

\}

voidaviso(double distancia)\{ //distância estabelecida para o acionamento dos

sensores. if (distancia $<=180 \& \&$ distancia $>=130)\{$

digitalWrite(buzz, HIGH);

digitalWrite(buzzdois, HIGH);

$\operatorname{delay}(700)$;

digitalWrite(buzz, LOW);

digitalWrite(buzzdois,

LOW); delay(600);

\}

else\{

if $($ distancia $<=129$

$\& \&$ distancia $>=90)\{$

digitalWrite(buzz, $\quad$ HIGH);

digitalWrite(buzzdois, $\quad$ HIGH);

delay(400);

digitalWrite(buzz, LOW);

digitalWrite(buzzdois,

LOW); delay(300); 


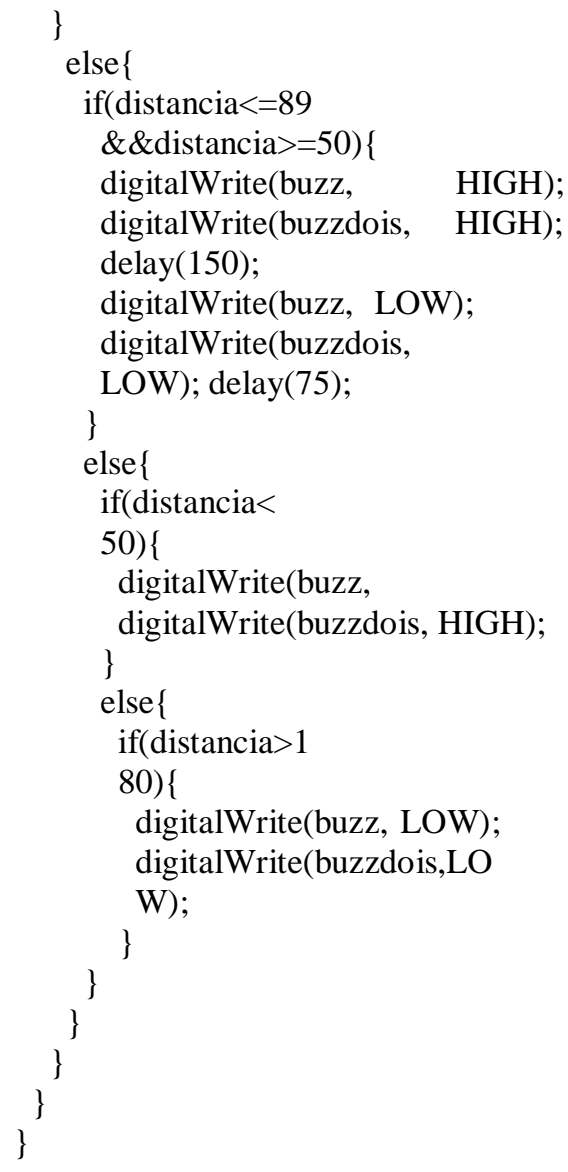

